\title{
Evolution of thrombectomy approaches and devices for acute stroke: a technical review
}

\author{
Alejandro M Spiotta, ${ }^{1}$ M Imran Chaudry, ${ }^{2}$ Ferdinand K Hui, ${ }^{3}$ Raymond D Turner, ${ }^{1}$ \\ Ryan T Kellogg, ${ }^{1}$ Aquilla S Turk ${ }^{2}$
}

${ }^{1}$ Division of Neurosurgery, Department of Neurosciences, Medical University of South Carolina, Charleston, South Carolina, USA

2Department of Neuroradiology, Medical University of South Carolina, Charleston, South Carolina, USA

${ }^{3}$ Cerebrovascular Center, Cleveland Clinic, Cleveland, Ohio, USA

\section{Correspondence to} Dr Alejandro M Spiotta, Division of Neurosurgery, Department of Neurosciences, Medical University of South Carolina, Charleston, SC 29425, USA;

spiotta@musc.edu

Received 23 October 2013 Revised 11 December 2013 Accepted 12 December 2013 Published Online First

2 January 2014

\section{ABSTRACT}

While intravenous administration of tissue plasminogen activator (tPA) remains the only FDA-approved treatment modality for acute ischemic stroke, many patients do not meet the criteria for intravenous tPA and are offered intra-arterial therapy. Rapid advances in devices and approaches have marked the evolution of thrombectomy over the past decade from rudimentary mechanical disruption, followed by intra-arterial thrombolytic infusions to increasingly effective thrombectomy devices. We review the critical advancements in thrombectomy technique that have evolved and the key anatomic and technical challenges they address, from first-generation Merci retrieval systems to second-generation Penumbra aspiration systems and third-generation stent retrievers, as well as nuances of their uses to maximize their effectiveness. We also highlight more recent advances that offer patients hope for more expedient vessel recanalization.

\section{INTRODUCTION}

Since its approval in 1995, the administration of systemic intravenous tissue plasminogen activator (tPA) is the only FDA-approved treatment modality for acute ischemic stroke, ${ }^{1}{ }^{2}$ despite rapid advances in thrombectomy devices. However, the restrictive time window after symptom onset (up to 3-4.5 h) and sociocultural and geographical barriers to accessing rapid care results in only a small minority of patients with ischemic stroke receiving treatment with intravenous tPA. ${ }^{3}{ }^{4}$ In addition, intravenous tPA has been demonstrated to be less effective in large vessel occlusions. ${ }^{5}$ Intra-arterial techniques were attempted to treat large vessel occlusions, initially using urokinase and prourokinase as described in the PROACT I and II trials, ${ }^{6} 7$ which was followed by the development of devices designed for intra-arterial thrombectomy and thromboaspiration. Although recent trials have failed to show a benefit over intravenous tPA of intra-arterial intervention using available devices, ${ }^{89}$ patients outside the intravenous tPA window are still considered candidates for intra-arterial procedures.

Acute stroke thrombectomy approaches have evolved rapidly. Spurred primarily by advances in catheter technology as well as the thrombectomy device itself, we are now able to achieve higher recanalization rates than ever before. We review the key technological advances and design modifications that have allowed for navigation around the ophthalmic turn for more distal delivery of larger- bore catheters providing more aspiration force directly applied at the thrombus interface.

\section{INTRA-ARTERIAL THROMBOLYSIS}

Historically, the mainstay of intra-arterial therapy for clot lysis in acute ischemic stroke was the administration of a thrombolytic agent into the vessel of interest. $^{6} 1011$ The PROACT II study was a randomized trial of intra-arterial infusion of recombinant prourokinase (r-proUK) versus placebo (heparinized saline) in patients with angiographically documented proximal middle cerebral artery occlusion. ${ }^{7}$ Thrombolytic infusion was associated with significantly higher recanalization rates and improved patient outcomes with acceptable complication rates. ${ }^{6} 7$ Despite this significant difference, the FDA did not approve prourokinase for this indication. Local thrombolytic infusions have been performed since the PROACT II study in 'off-label' fashion to treat both anterior and posterior circulation occlusions. ${ }^{4}$ 10-13 In addition, some operators employed intra-arterial infusions of tPA or abciximab to further promote clot lysis. ${ }^{14}$

\section{Adjunctive endovascular thrombectomy attempts}

To overcome the limitations of intra-arterial thrombolytic infusions alone, neurointerventionalists initially began attempting clot disruption with microwire manipulation. Shaping the microwire into a ' $\mathrm{J}$ ' or ' $\mathrm{C}$ ' shape and repeatedly advancing it through the thrombus was sometimes successful at recanalizing an occluded vessel. For more aggressive attempts, the microcatheter could be repeatedly advanced through the thrombus while leaving the microwire purchased in the distal vasculature to 'plow' through the thrombus. Early methods of thrombectomy included use of the goose-neck snare for clot capture and removal.

In 2005, flexible intracranial balloon catheters were introduced. They were initially designed for vessel angioplasty and later for balloon remodeling during aneurysm coil embolization. Shortly thereafter, these balloons were used to achieve mechanical clot disruption by repeated angioplasty of the thrombus itself. ${ }^{15}$

The introduction of intracranial stents presented another potential tool to achieve thrombectomy. ${ }^{16}$ The Enterprise vascular reconstructive device (Codman, Raynham, Massachusetts, USA) is a retrievable closed-cell design stent that could be partially deployed within the segment of the occluded vessel to achieve both mechanical disruption and partial flow restoration without committing to permanent stent deployment. ${ }^{17} 18$ Some operators have 
deployed intracranial stents in the occluded vessel, even in cases not involving underlying intracranial stenosis. ${ }^{19} 20$ The SARIS trial $^{1821}$ was an FDA-approved pilot study of stent placement within occluded vessels in acute stroke that showed high recanalization rates and good functional outcomes; however, the technique is limited by the need for dual antiplatelet therapy.

All of these devices were used in acute ischemic stroke in an 'off-label' fashion, not primarily designed for stroke intervention. However, these devices and uses set the stage for the design and development of dedicated devices. The following section outlines the various approaches, with a recognition that iterative improvements of each approach continue to be made.

\section{EVOLUTION OF APPROACHES TO TREATMENT OF ACUTE STROKE}

\section{The first device: the Merci retriever}

In 2004 the Merci retriever (Concentric Medical, Mountain View, California, USA) became the first mechanical thrombectomy device cleared for human use in the USA by the FDA. ${ }^{22}$ The Merci device primarily works by engaging the thrombus with a 'corkscrew' distal wire and suture tip deployed from within the clot, then removing the thrombus en bloc to achieve recanalization. The device itself is delivered within a microcatheter $(18 \mathrm{~L}$, Concentric Medical). The original iterations of the Merci X series included the 2.5, 3.0 and $3.5 \mathrm{~mm}$ diameters. Later the $\mathrm{L}$ series was introduced in 2006 and the $\mathrm{V}$ series in 2008. All these systems were employed using a balloon guide catheter that was positioned at the carotid bifurcation. Balloon inflation causes temporary flow reversal, allowing the Merci to be retrieved into the guide while mitigating the possibility of emboli showering to distal territories. However, clot retrieval into the guide catheter still required a long distance to be traveled while maintaining purchase on the thrombus, most commonly from the M1 segment of the middle cerebral artery to the proximal cervical internal carotid artery. Not only was this a long distance to travel, but also the vector force applied while pulling on the thrombus was suboptimal (downwards along the long axis of the cervical carotid artery, not horizontally along the axis of the middle cerebral artery). This caused considerable torqueing of the parent vessel and presented a biomechanical disadvantage to thrombus removal.

Revascularization rates in the Merci studies range from 43\% to $55 \% .^{23-25}$ However, thrombectomy with the Merci device as frontline therapy was not associated with a higher percentage of good functional outcomes (defined as modified Rankin Scale score $\leq 2$ at 90 days), reported in up to $36 \%$ of patients. ${ }^{25}$ Recanalization rates with the Merci system are thought to have increased since the initial trial from design refinements as well as increased operator experience. ${ }^{26}$

A landmark advancement came in 2010 with the approval of the Outreach Distal Access Catheter (DAC; Concentric Medical), which would have repercussions for the application of the Merci device and also for future iterations of thrombectomy approaches. The DAC was designed for the purpose of buttressing access for the Merci thrombectomy device, affording stable access to the target vessel. Use of the DAC optimized the vectors at play during pulling of the device. With further understanding of clot fragmentation and distal embolization, the DAC was used as an intermediate aspiration device which aided in preventing showering of distal emboli during clot retrieval, increased the aspiration power applied directly to the thrombus. ${ }^{27} 28$ The development of large-bore flexible catheters that could be delivered into the intracranial circulation represented a major advancement in thrombectomy technology and also in intermediate catheter technology. ${ }^{28} 29$ The DAC has a flexible distal shaft with increased proximal shaft strength and axial load-bearing characteristics as well as good hoop strength, allowing it to be delivered to the intracranial circulation around the ophthalmic bend when navigated over a coaxial catheter system. A major drawback to the Merci retrieval system was that it necessitated navigating past the ophthalmic bend with every pass, decreasing the efficiency of the system and adding to procedure times.

\section{The EKOS MicroLysUS catheter}

The EKOS microcatheter system (Bothwell, Washington, USA) and family of devices were designed to provide ultrasonic vibration to facilitate thrombolysis. Earlier studies employed a $2.5 \mathrm{~F}$ infusion catheter with an ultrasound-generating $2 \mathrm{~mm}$ transducer ring. ${ }^{30}$ The current EndoWave System (Bothwell) is a 5.2 F $106 \mathrm{~cm}$ long device using ultrasonic waves delivered via core wire and designed to be used in conjunction with intra-arterial tPA. ${ }^{31}$ Data regarding this device remain limited, but further development and experience may prove the approach beneficial.

\section{Second generation: Penumbra aspiration system}

The Penumbra aspiration system introduced in 2008 involves maceration of the thrombus with a separator which is repeatedly introduced and withdrawn from the thrombus under direct aspiration to prevent showering of fragments. ${ }^{32}$ While the Merci system relied primarily on a delivery microcatheter (the $18 \mathrm{~L}$; Stryker, Kalamazoo, Michigan, USA) to the site of occlusion, the Penumbra aspiration system relied on the delivery of a relatively large-bore catheter to the thrombus which ranges up to effectively a $5 \mathrm{~F}$ device. The introduction of highly-flexible lubricious polymers with good hoop strength allowed for placement of large intermediate class catheters directly into the circle of Willis. While the later introduction of the DAC catheter resulted in similar catheter profiles in the distal vasculature, the development of large-bore flexible catheters was essential to the function of the Penumbra system.

The original iteration of the Penumbra reperfusion catheter system included several different sized catheters (internal diameter 026, 032, and 041) and accompanying separators to maximize clot interaction and force of aspiration in vessels of differing diameters (internal carotid artery terminus, M1, M2, M3) to address both proximal and distal thrombi. ${ }^{33}$ The largest device had a lumen diameter of 0.041 inch and it tracked suboptimally and required a median $45 \mathrm{~min}$ to achieve acceptable recanalization. In 2009 the Reperfusion Catheter 054 became available which dramatically improved the aspiration efficiency to a median $20 \mathrm{~min}^{34}$ due to its much larger tapered lumen. The aspiration force is proportional to the square of the diameter of the catheter, so the 054 catheter provided an estimated $4 \times$ aspiration force over the next smaller catheter, the $041 .^{34}$

Despite the improved technology, the 054 still required a coaxial catheter for delivery to the middle cerebral artery. Although a larger catheter lumen provides higher suction and more rapid removal of material, it also results in a larger catheter profile and more difficult distal navigation. Owing to its size, the 054 catheter often required the use of a coaxial technique to facilitate navigation to the site of occlusion. When navigated over a 0.014 inch microwire alone, a significant ledge would get held up at the origin of the ophthalmic artery. To overcome this obstacle to the target lesion, access with the 054 catheter can be optimized with a coaxial technique (figure 1). The smaller 032 and 026 reperfusion catheters can be delivered simply over either a 0.014 or 0.016 inch wire and the larger 

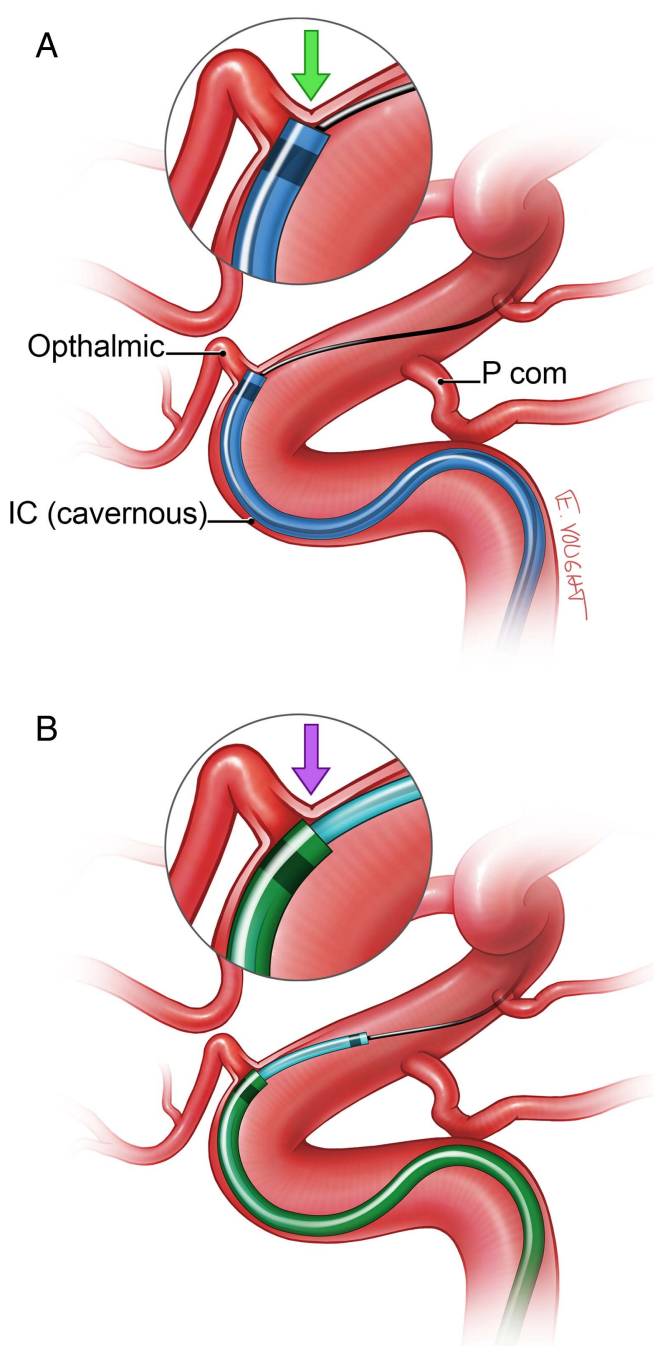

Figure 1 (A) While a larger catheter lumen provides higher suction and more rapid removal of thrombus, it results in a larger catheter profile and 'ledge effect' which renders navigation past the ophthalmic artery origin challenging. (B) To overcome this obstacle, access with an intermediate catheter is optimized with a coaxial technique resulting in a more tapered construct that minimizes the 'ledge effect'.

054 delivered over those. One of the major advantages of the Penumbra aspiration system was that, once the catheter system was delivered to the target vessel, separator clot maceration could be performed without having to re-access (additional 'passes'), as was the case for the Merci device. ${ }^{35}$

Despite these advances in catheter technology, navigating past the carotid siphon was still a relative challenge to thrombectomy cases. In patients with very acute angulation in the ophthalmic segment, adjunctive techniques could be performed to obtain the necessary distal access. One approach used the Merci retriever system as an adjunct to improve the trackability of the 054 reperfusion catheter by altering the angle with which the catheter engages the ophthalmic segment and M1 origin. By deploying an appropriately-sized Merci Retriever (Concentric Medical), such as a V.2.0 or V.2.5 soft, in the mid M1 segment through either the 032 catheter or an $18 \mathrm{~L}$ microcatheter and then applying gentle traction on the Merci Retriever, the course of the wire straightens, approximating the inner curve of the vasculature, pulling the catheter complex away from the ledge of the vessel origins ('grappling hook' technique), 2836 an approach now used routinely using stent retrievers and intermediate catheters. The 054 catheter can then be more readily advanced into the target vessel. Once the 054 reperfusion catheter is in place, the retriever is resheathed into the $18 \mathrm{~L}$ microcatheter and then removed prior to separator placement and aspiration.

The next iteration of the Penumbra aspiration catheter family (Max series) was introduced in 2012 and included larger inner diameters at the distal end as well as the proximal end to increase the aspiration power. The larger proximal lumen reduces resistance to flow and therefore increases aspiration force at the catheter tip. Improvements in polymer and braid/ ring reinforcement provide more catheter tip flexibility and an increased number of transition zones to improve trackability. The newly introduced intermediate catheters were named 5Max, 4Max and 3Max. An increased number of transition zones in the catheter design and manufacturing allowed these catheters to be delivered primarily over either a 0.014 or 0.016 inch microwire, even past the ophthalmic origin.

\section{Third generation: stent retrievers}

The next generation of mechanical thrombectomy devices includes the 'stent retriever' family: Solitaire (ev3 Endovascular, Plymouth, Minnesota, USA), Trevo Pro (Stryker Neurovascular, Kalamazoo Michigan, USA) and arguably the Penumbra 3D separator (Penumbra, Alameda, California, USA)). The formal stent retrievers are literally stents that are fully recapturable and fused to the delivery microwire. Differences in cell design and lubricity and end portion variations have also been introduced in pre-market stent retrievers and are beyond the scope of this article. The Penumbra 3D separator differs from formal stent retrievers in that there is no stent. A larger portion of the separator design by mass is designed to engage at the center of the vessel lumen rather than in an actual stent where the material is primarily at the outer margins of the vessel near the intima. These devices have the advantage of efficacious recanalization like a stent but are able to be removed, which obviates the need for clopidogrel and aspirin. While the Solitaire was the first to be released, all major device manufacturers produce similar devices. ${ }^{37}$ Stent retrievers capitalize on the advantages observed with partial stent deployment and recapture during thrombectomy. The microcatheter is delivered across the thrombus and the stent retriever is unsheathed from within the thrombus itself. The outward radial force of the stent retriever as it deploys promotes engaging the clot. While doing so, cerebral blood flow to the involved territory is temporarily restored, functioning as an 'endovascular bypass'. Once the stent retriever has engaged the thrombus, it is pulled back ('retrieved') into a guide catheter. Application of suction with either a pump or manual syringe aspiration during retrieval may promote clot purchase and reduce the showering of emboli.

To minimize the distance the stent retriever must travel while engaging the thrombus, especially into larger caliber vasculature such as the internal carotid artery from the middle cerebral artery, and mitigating the possibility of losing purchase of the clot, variations to the stent retriever technique have been employed with incorporation of Penumbra reperfusion catheters. For example, a $5 \mathrm{Max}$ catheter can be advanced over an 025 microcatheter and microwire up to the site of occlusion and left at the face of the thrombus. The stent retriever is then deployed and the microcatheter is removed, leaving the stent retriever in place. The Stent retriever is then pulled directly into the 5Max while maintaining aspiration (so-called 'Solumbra' technique since it combines a stent retriever (Solitaire) with a 
Penumbra aspiration catheter), and both are removed together, much in the same way as the Merci retriever device was removed with a DAC. Recanalization rates with stent retrievers were found to be superior to the Merci device in several studies, ${ }^{37} 38$ leading to rapid and widespread adoption.

\section{New generation: direct aspiration}

Direct aspiration has become possible due to advances in catheter technology that allow large-caliber aspiration catheters to be advanced intracranially to the thrombus. In general, the largest size aspiration catheter that the vessel can accommodate should be used. At our institution this is most commonly a Penumbra 5Max reperfusion catheter (Penumbra, Oakland, California, USA) for M1 or carotid terminus occlusions. The 5 Max can be advanced to the level of the thrombus over any microcatheter and microwire the operator choses, but most commonly a Velocity microcatheter (Penumbra) over a 0.016 inch Fathom wire (Boston Scientific Corp, Naidich, Massachusetts, USA). The microcatheter and wire are removed and aspiration is applied by either a 20 or $60 \mathrm{~mL}$ syringe or use of the Penumbra aspiration pump that is part of the Penumbra thrombectomy/aspiration system. ${ }^{33}$ Inability to draw back on aspiration confirms the optimal position of the 5Max catheter abutting the thrombus. More recently, an alternative technique has been used where the catheter is slightly advanced to ensure firm engagement with the thrombus. The 5Max catheter is then slowly withdrawn while maintaining aspiration. Aspiration is also applied to the sideport of the guide catheter to prevent dislodging the thrombus from the 5Max aperture as it is withdrawn into the sheath. In smaller caliber vessels, the technique can be employed with either a 4Max or 3Max reperfusion catheter (Penumbra). When this technique is successful it eliminates the need to introduce stent retriever or Penumbra separator devices, leading to an overall much lower procedure device cost. $^{39}$ Thus, we have found the initial application of this technique to provide the highest cost-effective value in acute stroke treatment.

This approach was facilitated by the development of the Penumbra Max aspiration catheter technology which significantly increased the ease and speed of navigation of a large-bore catheter into the intracranial circulation. The direct aspiration technique differs from previous thrombectomy methods as it focuses on engaging and removing the clot in its entirety rather than the use of the separator that was designed to macerate the thrombus and clear the tip of the aspiration catheter. ${ }^{40}$ Historically, due to the challenges with tracking an aspiration catheter into the intracranial circulation, catheters had to be telescoped with other catheters together or other tricks employed to advance through the siphon. ${ }^{28} 29364142$ However, the superior trackability of the new trackable large-bore aspiration catheters (eg, Penumbra 5Max) has given us the confidence to attempt direct aspiration alone without the fear that it will be a significant time and danger impediment to the patient if intracranial access is lost. Perhaps most importantly, if aspiration

\section{Thrombectomy Device Timeline}

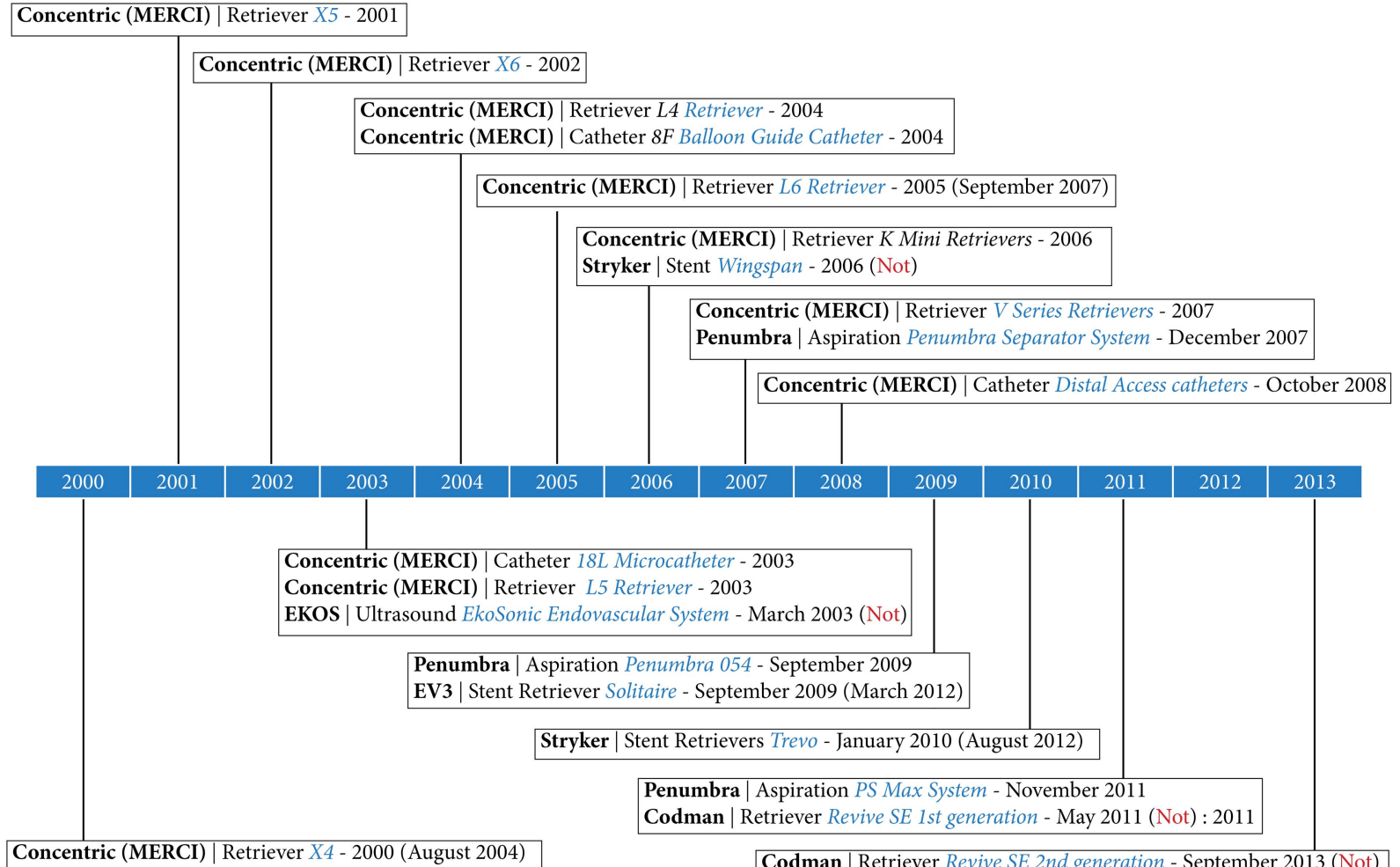

Concentric (MERCI) | Retriever X4 - 2000 (August 2004)

Figure 2 Timeline of the release dates of the major devices relating to stroke thrombectomy. 
Table 1 Comparison of the results of clinical trials employing different devices for mechanical thrombectomy for acute ischemic stroke

\begin{tabular}{lllll}
\hline & Merci & Penumbra & & \\
& $2002-$ & $2007-$ & SWIFT & TREVO \\
Trial & $2007^{12}$ & $2009^{3}$ & 2012 & $2012^{6}$ \\
\hline Patients & $151^{1}$ & $125^{3}$ & $89^{5}$ & $88^{6}$ \\
& $164^{2}$ & $157^{4}$ & & \\
Mean NIHSS & $20.1^{1}$ & $17.6^{3}$ & $17.4^{5}$ & $18.3^{6}$ \\
& $19^{2}$ & $16.6^{4}$ & & \\
Symptom onset to treatment (h) & $4.3^{1}$ & $4.3^{3}$ & $4.8^{5}$ & $4.6^{6}$ \\
& $4.3^{2}$ & $4.1^{4}$ & & \\
Treatment onset to recanalization & $126^{1}$ & $45^{3}$ & $36^{* 5}$ & $44^{* 6}$ \\
time (min) & $96^{2}$ & $41^{4}$ & & \\
Procedure complication (\%) & $13^{1}$ & $12.8^{3}$ & $15.7^{5}$ & $15^{6}$ \\
& $9.8^{2}$ & $5.8^{4}$ & & \\
Mortality at 90 days (\%) & $43.5^{1}$ & $33^{3}$ & $17^{5}$ & $33^{6}$ \\
& $34^{2}$ & $20^{4}$ & & \\
mRS $\leq 2$ at 90 days (\%) & $27.7^{1}$ & $25^{3}$ & $36^{5}$ & $40^{6}$ \\
& $36^{2}$ & $41^{4}$ & & \\
Recanalization rate (\%) & $48^{1}$ & $82^{3}$ & $61^{5}$ & $86.4^{6}$ \\
& $68^{2}$ & $87^{4}$ & & \\
\hline
\end{tabular}

* Recanalization time defined as the time from baseline guide catheter run to visualization of Thrombolysis In Myocardial Infarction (TIMI).

mRS, modified Rankin Scale; NIHSS, NIH Stroke Scale. alone is not successful at revascularizing the occluded vessel, the large-bore aspiration catheters can function as a direct distal conduit for other devices such as stent retrievers, balloons or stents.

The newest iteration, the 5Max ACE (introduced in June 2013) with 12 transition zones has an increased inner diameter of 0060 at the distal $30 \mathrm{~cm}$, while housing a 0.068 proximal end for larger aspiration forces. At the time of writing, early ADAPT experience with retrospective and prospective data is very encouraging and includes a patient sample size as large as the SWIFT and TREVO 2 trials (presented at the World Federation of Interventional and Therapeutic Neuroradiology, Buenos Aires, 2013).

\section{FUTURE DEVICES}

On-label intra-arterial devices for acute stroke intervention are developing rapidly with the introduction of both iterative changes and new classes of devices (figure 2). With new generations of devices, increasingly high rates of recanalization are being reported (table 1). It is likely that new techniques and devices will continue to evolve, offering a more robust tool set and combination of devices for interventional management of acute stroke. However, futile recanalization remains a problem. ${ }^{43}$ The development of ever more efficient intra-arterial

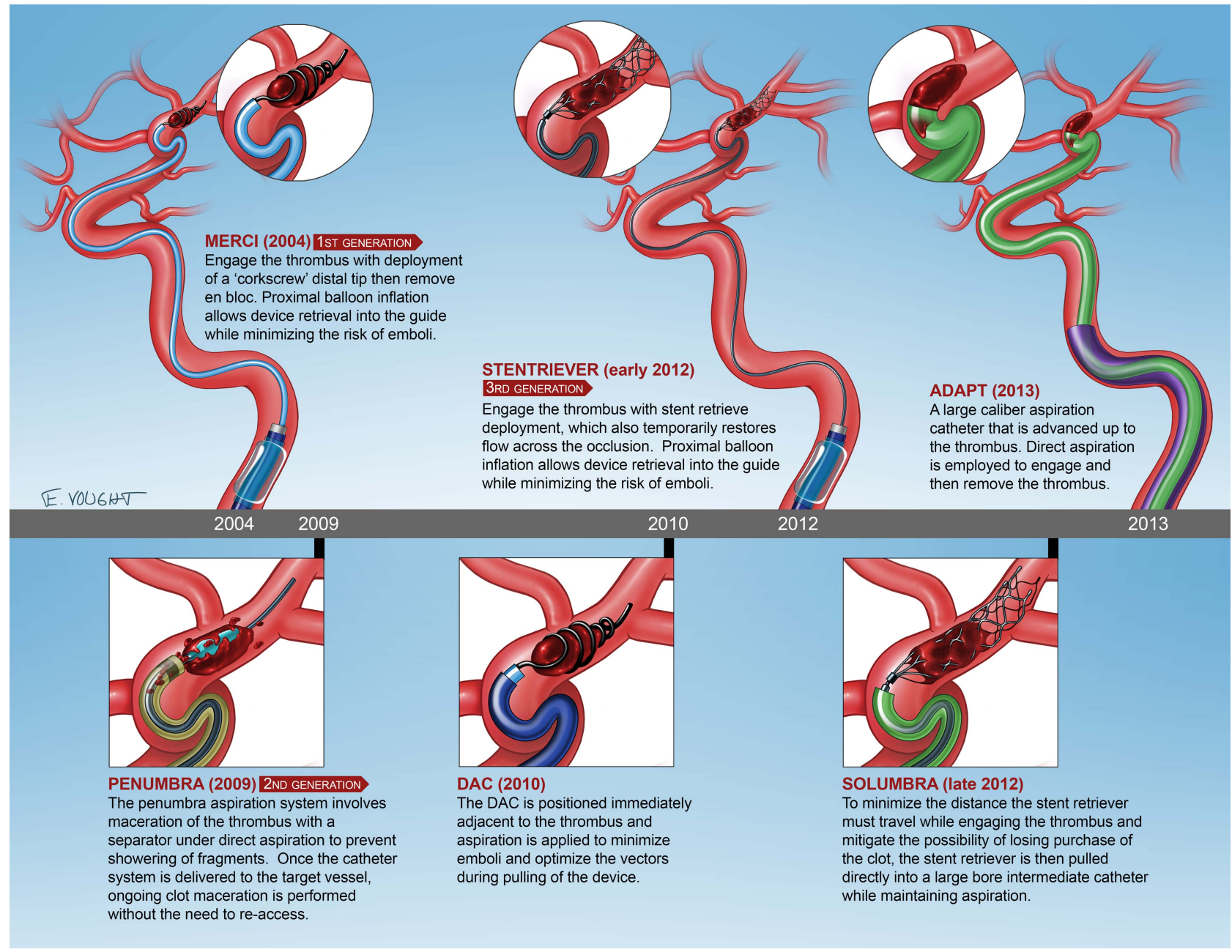

Figure 3 Illustration depicting the major steps in evolution of thrombectomy devices, beginning from the first-generation concept to state-of-the-art approaches. 
approaches may provide the appropriate interventional approaches to appropriately selected patients.

\section{CONCLUSIONS}

There have been rapid advances in thrombectomy devices and approaches over the past decade, from rudimentary mechanical disruption followed by intra-arterial thrombolytic infusions to increasingly effective thrombectomy devices (figure 3). While it remains unknown what combinations of techniques, devices, selection criteria and medicines will yield the best outcomes, ongoing improvements in the devices and techniques are yielding improved angiographic and clinical outcomes. Device technology, selection strategies and medical management will probably evolve in tandem, and we look forward to the continued evolution of thrombectomy approaches for acute stroke in the future. We remind our colleagues in the neurointerventional field that high enrollment in clinical trials will be required to secure the role of intra-arterial therapy in the management of stroke.

Contributors Each author made a material contribution to the article, its revision and the final approval of the article for submission.

Competing interests None.

Ethics approval Ethics approval was obtained from the Medical University of South Carolina IRB.

Provenance and peer review Not commissioned; externally peer reviewed.

\section{REFERENCES}

1 Anon. Tissue plasminogen activator for acute ischemic stroke. The National Institute of Neurological Disorders and Stroke rt-PA Stroke Study Group. N Engl J Med 1995;333:1581-7.

2 Jauch EC, Saver JL, Adams HP Jr, et al. Guidelines for the early management of patients with acute ischemic stroke: a guideline for healthcare professionals from the American Heart Association/American Stroke Association. Stroke 2013;44:870-947.

3 Fonarow GC, Smith EE, Saver JL, et al. Timeliness of tissue-type plasminogen activator therapy in acute ischemic stroke clinical perspective patient characteristics, hospital factors, and outcomes associated with door-to-needle times within 60 minutes. Circulation 2011;123:750-8.

4 Hirsch JA, Yoo AJ, Nogueira RG, et al. Case volumes of intra-arterial and intravenous treatment of ischemic stroke in the USA. J Neurointerv Surg 2009:1:27-31.

5 Wechsler LR, Roberts R, Furlan AJ, et al. Factors influencing outcome and treatment effect in PROACT II. Stroke 2003:34:1224-9.

6 del Zoppo GJ, Higashida RT, Furlan AJ, et al. PROACT: a phase II randomized trial of recombinant pro-urokinase by direct arterial delivery in acute middle cerebral artery stroke. PROACT Investigators. Prolyse in acute cerebral thromboembolism. Stroke 1998;29:4-11.

7 Furlan $A$, Higashida $\mathrm{R}$, Wechsler $\mathrm{L}$, et al. Intra-arterial prourokinase for acute ischemic stroke. JAMA 1999;282:2003-11.

8 Broderick JP, Palesch YY, Demchuk AM, et al. Endovascular therapy after intravenous t-PA versus t-PA alone for stroke. N Engl J Med 2013;368:893-903.

9 Chimowitz MI. Endovascular treatment for acute ischemic stroke-still unproven. N Engl J Med 2013;368:952-5.

10 Ernst R, Pancioli A, Tomsick $T$, et al. Combined intravenous and intra-arterial recombinant tissue plasminogen activator in acute ischemic stroke. Stroke 2000;31:2552-7

11 Suarez J, Sunshine J, Tarr R, et al. Predictors of clinical improvement, angiographic recanalization, and intracranial hemorrhage after intra-arterial thrombolysis for acute ischemic stroke. Stroke 1999:30:2094-100.

12 Brandt $T$, von Kummer R, Muller-Kuppers $M$, et al. Thrombolytic therapy of acute basilar artery occlusion. Variables affecting recanalization and outcome. Stroke 1996;27:875-81.

13 Hacke W, Zeumer $\mathrm{H}$, Ferbert A, et al. Intra-arterial thrombolytic therapy improves outcome in patients with acute vertebrobasilar occlusive disease. Stroke 1988; 19:1216-22.

14 Abou-Chebl A, Bajzer CT, Krieger DW, et al. Multimodal therapy for the treatment of severe ischemic stroke combining GPIIb/llla antagonists and angioplasty after failure of thrombolysis. Stroke 2005:36:2286-8.

15 Ringer AJ, Qureshi Al, Fessler RD, et al. Angioplasty of intracranial occlusion resistant to thrombolysis in acute ischemic stroke. Neurosurgery 2001;48:1282-90.
16 Levy El, Siddiqui AH, Crumlish A, et al. First Food and Drug Administration-approved prospective trial of primary intracranial stenting for acute stroke SARIS (stent-assisted recanalization in acute ischemic stroke). Stroke 2009;40:3552-6.

17 Kelly ME, Furlan AJ, Fiorella D. Recanalization of an acute middle cerebral artery occlusion using a self-expanding, reconstrainable, intracranial microstent as a temporary endovascular bypass. Stroke 2008;39:1770-3.

18 Levy El, Rahman M, Khalessi AA, et al. Midterm clinical and angiographic follow-up for the first food and drug administration-approved prospective, single-arm trial of primary stenting for stroke: saris (stent-assisted recanalization for acute ischemic stroke). Neurosurgery 2011;69:915-20.

19 Levy El, Ecker RD, Horowitz MB, et al. Stent-assisted intracranial recanalization for acute stroke: early results. Neurosurgery 2006;58:458-63.

20 Mocco J, Hanel RA, Sharma J, et al. Use of a vascular reconstruction device to salvage acute ischemic occlusions refractory to traditional endovascular recanalization methods: clinical article. J Neurosurg 2010;112:557-62.

21 Roth C, Papanagiotou P, Behnke S, et al. Stent-assisted mechanical recanalization for treatment of acute intracerebral artery occlusions. Stroke 2010;41:2559-67.

22 Gobin YP, Starkman S, Duckwiler GR, et al. MERCI 1: a phase 1 study of mechanical embolus removal in cerebral ischemia. Stroke 2004;35:2848-54.

23 Flint AC, Duckwiler GR, Budzik RF, et al. Mechanical thrombectomy of intracrania internal carotid occlusion: pooled results of the MERCI and Multi MERCI Part I trials. Stroke 2007:38:1274-80.

24 Smith W. Safety of mechanical thrombectomy and intravenous tissue plasminogen activator in acute ischemic stroke. Results of the multi Mechanical Embolus Removal in Cerebral Ischemia (MERCI) trial, part I. Am J Neuroradiol 2006;27:1177-82.

25 Smith WS, Sung G, Starkman S, et al. Safety and efficacy of mechanical embolectomy in acute ischemic stroke results of the MERCI trial. Stroke 2005;36:1432-8.

26 Smith WS, Sung G, Saver J, et al. Mechanical thrombectomy for acute ischemic stroke final results of the multi MERCI trial. Stroke 2008;39:1205-12.

27 Jankowitz B, Aghaebrahim A, Zirra A, et al. Manual aspiration thrombectomy adjunctive endovascular recanalization technique in acute stroke interventions. Stroke 2012:43:1408-11.

28 Spiotta AM, Hussain MS, Sivapatham T, et al. The versatile distal access catheter: the Cleveland Clinic experience. Neurosurgery 2011;68:1677.

29 Turk A, Manzoor MU, Nyberg EM, et al. Initial experience with distal guide cathete placement in the treatment of cerebrovascular disease: clinical safety and efficacy. J Neurointerv Surg 2013;5:247-52.

30 Mahon BR, Nesbit GM, Barnwell SL, et al. North American clinical experience with the EKOS MicroLysUS infusion catheter for the treatment of embolic stroke. Am J Neuroradiol 2003;24:534-8.

31 Kuliha M, Roubec M, Fadrná T, et al. Endovascular sono-lysis using EKOS system in acute stroke patients with a main cerebral artery occlusion-a pilot study. Perspect Med 2012;1:65-72.

32 Clark W, Lutsep $H$, Barnwell S, et al. The penumbra pivotal stroke trial: safety and effectiveness of a new generation of mechanical devices for clot removal in intracranial large vessel occlusive disease. Stroke 2009;40:2761-8.

33 Yoo AJ, Frei D, Tateshima S, et al. The Penumbra Stroke System: a technical review. J Neurointerv Surg 2012;4:199-205.

34 Frei D, Gerber J, Turk A, et al. The SPEED study: initial clinical evaluation of the Penumbra novel 054 reperfusion catheter. J Neurointerv Surg 2013;5(Suppl 1): i74-6.

35 Tarr R, Hsu D, Kulcsar Z, et al. The POST trial: initial post-market experience of the Penumbra system: revascularization of large vessel occlusion in acute ischemic stroke in the United States and Europe. J Neurointerv Surg 2010:2:341-4.

36 Hui FK, Hussain MS, Spiotta A, et al. Merci retrievers as access adjuncts for reperfusion catheters: the grappling hook technique. Neurosurgery 2012;70:456-60.

37 Saver JL, Jahan R, Levy El, et al. Solitaire flow restoration device versus the Merci Retriever in patients with acute ischaemic stroke (SWIFT): a randomised, parallel-group, non-inferiority trial. Lancet 2012;380:1241-9.

38 Nogueira RG, Lutsep HL, Gupta R, et al. Trevo versus Merci retrievers for thrombectomy revascularisation of large vessel occlusions in acute ischaemic stroke (TREVO 2): a randomised trial. Lancet 2012;380:1231-40.

39 Turk AS, Campbell JM, Spiotta A, et al. An investigation of the cost and benefit of mechanical thrombectomy for endovascular treatment of acute ischemic stroke. J Neurointerv Surg 2014;6:77-80.

40 Turk AS, Spiotta A, Frei D, et al. Initial clinical experience with the ADAPT technique: a direct aspiration first pass technique for stroke thrombectomy. J Neurointerv Surg 2014;6:231-7.

41 Chaudhary N, Pandey AS, Thompson BG, et al. Utilization of the Neuron 6 French 0.053 inch inner luminal diameter guide catheter for treatment of cerebral vascular pathology: continued experience with ultra distal access into the cerebral vasculature. J Neurointerv Surg 2012;4:301-6.

42 Park MS, Stiefel MF, Fiorella D, et al. Intracranial placement of a new, compliant guide catheter: technical note. Neurosurgery 2008;63:E616-17.

43 Molina CA. Futile recanalization in mechanical embolectomy trials a call to improve selection of patients for revascularization. Stroke 2010:41:842-3. 